\section{EPiC Series in Health Sciences}

Volume 1, 2017, Pages 407-416

CAOS 2017. 17th Annual Meeting of the International

Society for Computer Assisted Orthopaedic Surgery
EPIC

Health

Sciences

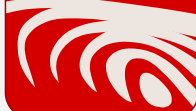

\title{
PSI for Dog Knee Replacement
}

Mahmoud A. Hafez ${ }^{1}$, Ahmed Abdel Moghny ${ }^{1}$ and Ashraf Shamaa ${ }^{2}$

${ }^{1}$ The Orthopedic Dept., October 6 University, Giza, Egypt

${ }^{2}$ Surgery and Radiology Dept., Faculty of Veterinary Medicine, Cairo

University, Cairo, Egypt.

\begin{abstract}
Total knee replacement (TKR) is a surgical interference in advanced knee osteoarthritis or traumatic injury. Patient-specific instrument (PSI) for TKR is a computer-assisted orthopaedic surgery (CAOS) which relies on preoperative planning on CT or MRI. The introduction of computer technology in orthopaedics did not only allow for the production of PSI but also customized implants. In this work, we demonstrate a TKR procedure for dog that was done by PSI and customized implants.
\end{abstract}




\section{Introduction:}

The knee joint is a complex joint and is considered the most stressed joint in the body [1, 2]. The canine's stifle is closely matched to the human knee in both macroscopic and microscopic anatomy apart from the size, the intraarticular long digital extensor tendon and the lateral and medial sesamoids in the head of the gastrocnemius muscle [3]. TKR was developed to address arthritic conditions and degenerative joint diseases which involve joint capsule synovium, articular cartilage and subchondral bone as well as bony over growth [4-6].

Computer-assisted orthopaedic surgical (CAOS) techniques such as robotics and navigation started in the 1980s with several neurosurgical applications. The technology transferred from neurosurgery to orthopaedics for spine, hip and knee surgery. It developed from active robotics navigation systems such as navigation by intraoperative fluoroscopy or image-free [7, 8].

Canine knee arthroplasty was experimented to study of bone ingrowth with porous coated stainless steel hinged knee implants [9]. This experiment was further detailed by using unconstrained condylar prosthesis with a wide bearing surface and single radius of curvature on both the femoral and tibial components [10]. Afterwards, the third generation of canine's implant was developed by contour-based geometry, that is, the articulating surface of the implants was replicated from radiograph obtained femoral contour [11].

The aim of this work is to demonstrate the applicability of PSI and customized implants in canine's TKR. 


\section{Methods:}

CT-scanning was done according to pre-defined protocol. DICOM file is transferred to an image-rocessing software (OrthoTaix) to start 3D planning. A virtual 3D model is constructed on bone and mechanical axis and rotations are defined. The designing step involves planning for surgical cuts, creating PSI and customized implants (Figures 1-7).

PSI is fabricated from plastics with additive manufacturing (3D printing) technique, while customized implants are fabricated of stainless steel and coated by Ti alloys (ti6al4v) with CNC technique; the material of tibial component is polyethylene (Figures 7-10). The templates as well as the implant were finished, packed and sterilized prior to surgery.

\section{Results:}

Knee prostheses were fabricated in a universal open-platform fashion that matched to the exact anatomical structure of the stifle. No need for coupling with particular drills, sleeves or surgical instruments that relate to a particular company. The same technique can be applied for other knee procedures such as unicompartmental, bicondylar and patellofemoral arthroplasty and in treating non-straight-forward cases.

\section{Discussion:}

In this work, preparation of an artificial knee joint for a canine's TKR surgery was feasible. A complete virtual surgery of TKR could be practiced a priori. PSI is composed of femoral and tibial parts, which were designed as complete cutting guides that match the articulating surfaces of the bone. 
Surface matching is guided intraoperatively by anatomical landmarks on bone surfaces.

The current technology of knee implants is based on planning TKA according to specific implant criteria (size and shape) so that the implant of one company cannot be used for implants of other companies. In turn, the currently used knee implants are expensive and company specific, which is a significant disadvantage and limits the wide-spread application of current availability of TKA. In addition, a serious limitation of the current TKA technique is that the planning is done by technicians not by surgeons, and the whole process in under the control of the implant company.

\section{Conclusion:}

3D technology was thus suitable for TKR for different breeds of dogs regardless of their size or morphology. This technology can replace the conventional instruments for TKA such as intramedullary, extra-medullary guides, sizing, rotation guides for both tibial and femur. The femoral implant did not extend to the trochlea; it had good anterior pegs and central stem.

\section{Disclosure:}

None.

\section{References:}

1. Bobyn, J "Determination of criteria for the design and application of porous-surfaced surgical implant” (1980), Ph.D. Thesis, University of Toronto. 
2. Buckwalter,A., Saltzman, C., Brown, T “The impact of osteoarthritis” (2004). Clin Orthoped Rel Res 2004:427S: S6-S15.

3. Catriona, M “Treatment of canine osteoarthritis” (2000), Colorado State University, WALTHAM Focus, vol 10 No 2.

4. Charles, D., David, M “Part XII: ARTHRITIS” Chapter 87: Degenerative Joint Disease and Traumatic Arthritis, "Textbook of Small Animal Orthopaedics"(1985).

5. Christopher, S., William, D “Custom Knee replacement for gunshot wound victum. M” (2004).BioMedtrix, USA

6. Duchenye, P., DeMester, P., Aernoudt, D “Influence of a functional dynamic loading on bone ingrowth into surface pores of orthopedic implants” (1977), Journal of biomedical materials Research 11:811-838.

7. Lyon, L “Canine and feline Anesthesia” (2006), Veterinary Surgery I, VMED 7412.

8. Martin, G., Nicholas, C., Keiichi, K., Aaron, S., James, c., Seth, S “A Review of Translational Animal Models for Knee Osteoarthritis” (2012), Volume 2012, article ID 764621.

9. Miller, W., Scott, D., Wellington, R “Treatment of dogs with hip arthritis with a fatty acid supplement” (1992), Canine Practice 1992; 17: 6-8.

10. Nicole, $\mathrm{H}^{\prime}$ Biomechanical analysis of the effects of a total knee replacement on canine stifle” (2011), University of Georgia.

11. Turner, T., Urban, R., Sumner, D., Skipor, A., Galante, J “Bone Ingrowth into the tibial Component of canine total condyler knee replacement prosthesis” (1989), Journal of orthopedic research, vol 7 No.6: 893-901. 
Figures:

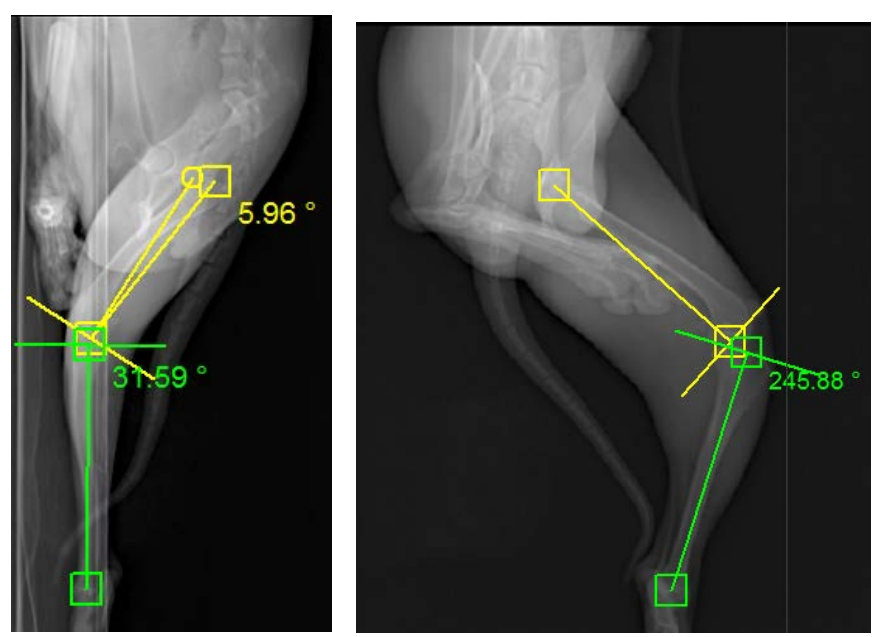

Figure (1): mechanical axis of Lt-Knee

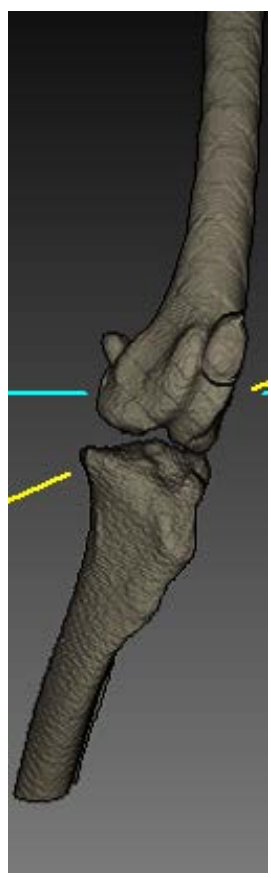

Figure (2): 3D bone reconstruction form capture CT-scan images. 

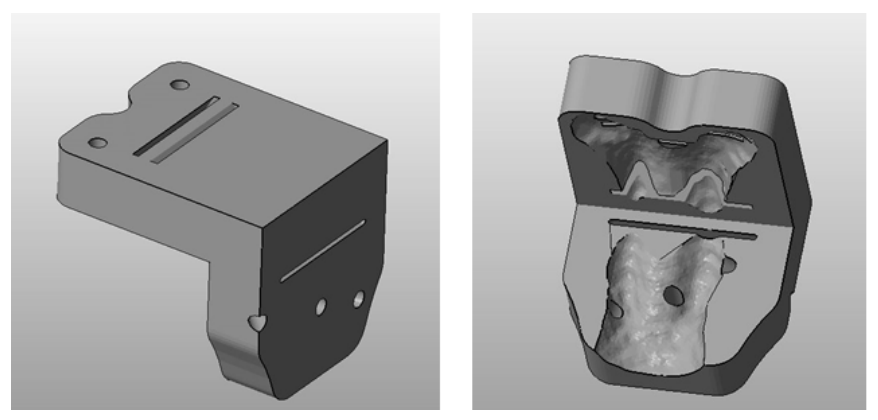

Figure (3): patient specific template, femoral component of $1^{\text {st }}$ version include three femoral cutting slots with multi holes for fixation on the bone surface.
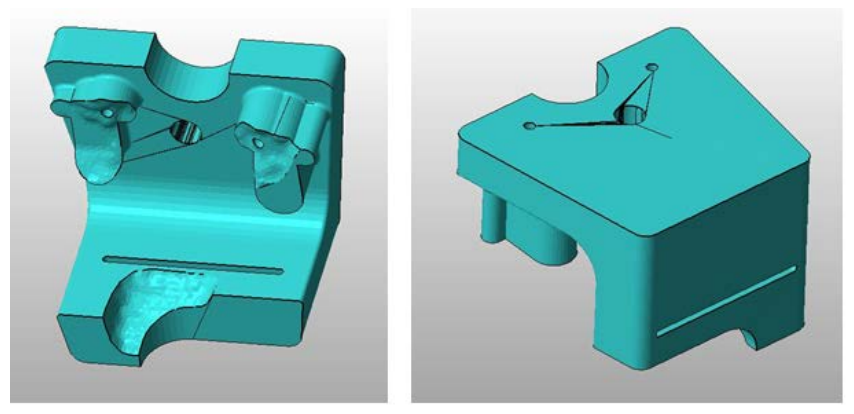

Figure (4): patient specific template, tibial component of $1^{\text {st }}$ version include tibial cutting slots with multi holes for fixation on the bone surface.
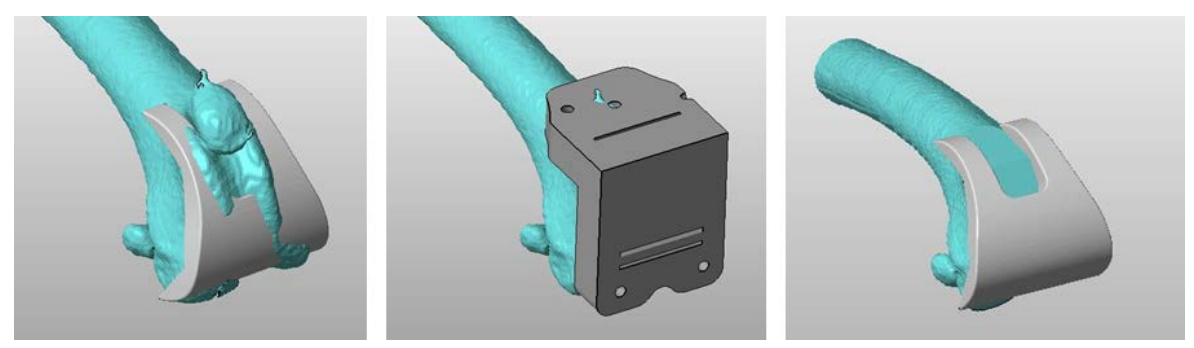

Figure (5): custom made implant (femoral component) positioning on the bone surface before and after cut. 

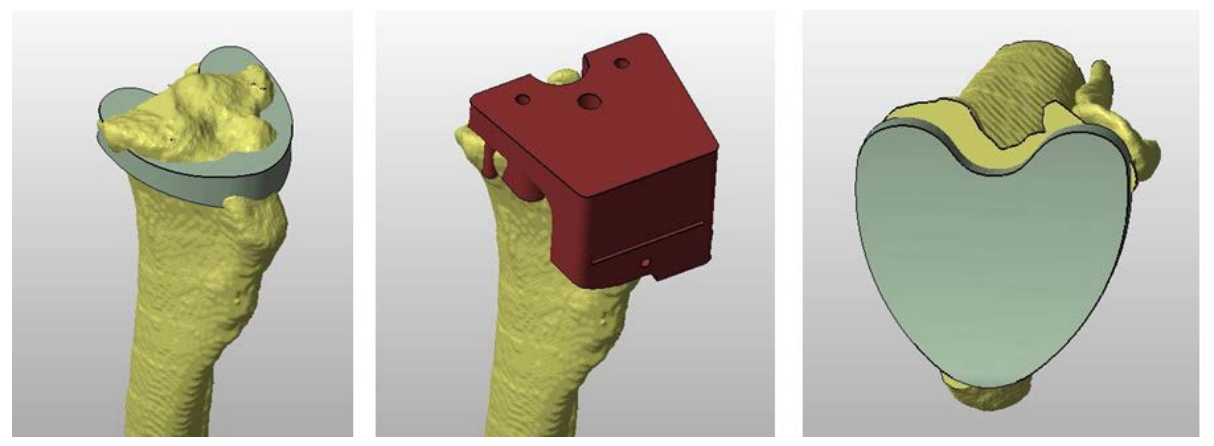

Figure (6): custom made implant (tibial component) positioning on the bone surface before and after cut. Patient specific template (tibial component) positioning on the bone surface.

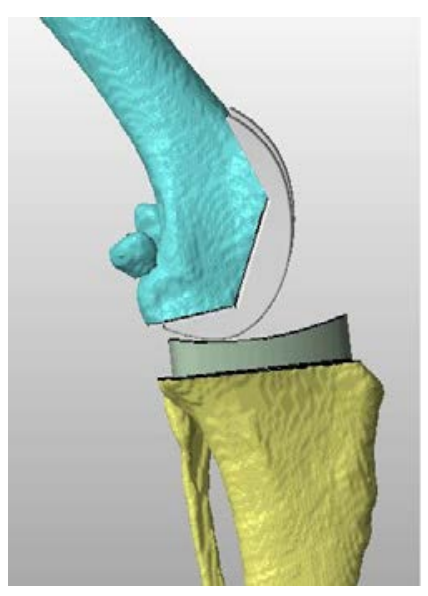

Figure (7): custom made implant in final position as planned for surgery. 

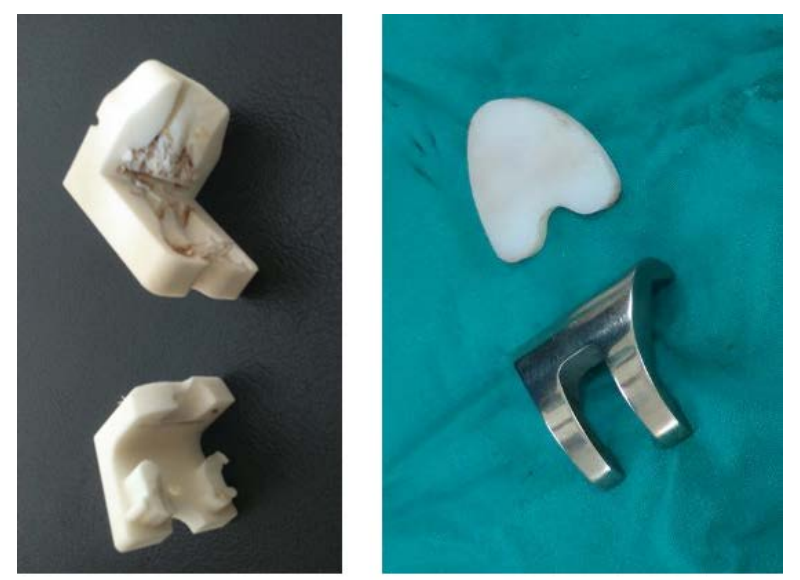

Figure (8): 3D printed custom made implant and patient specific templates.
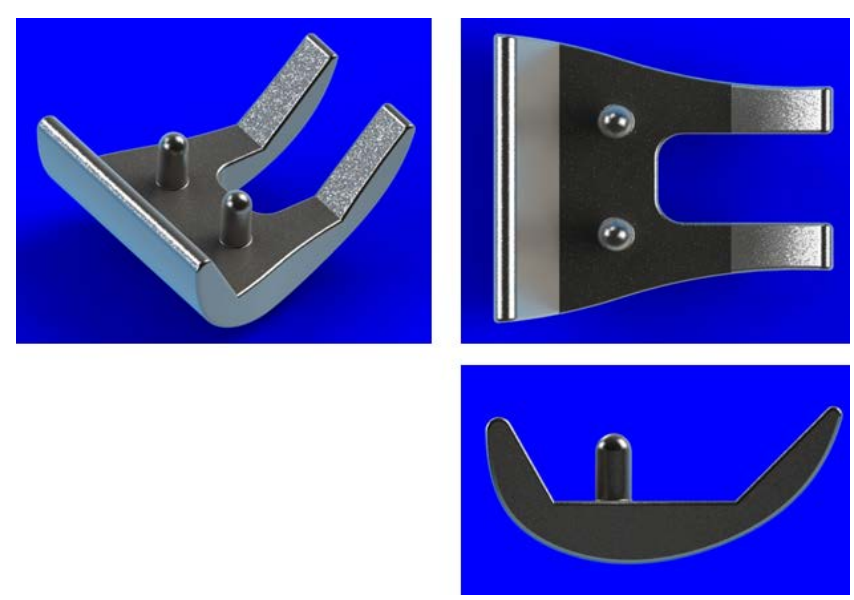

Figure (9): custom made implant, femoral component of 1st version include three femoral cut with two fixation pegs. 

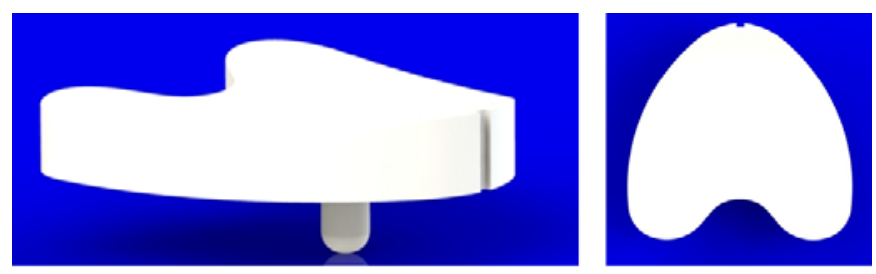

Figure (10): custom made implant, tibial component of $1^{\text {st }}$ version, designed as a convex shape with $6 \mathrm{~mm}$ thickness and $3.5 \mathrm{~mm}$ diameter of a central stem.
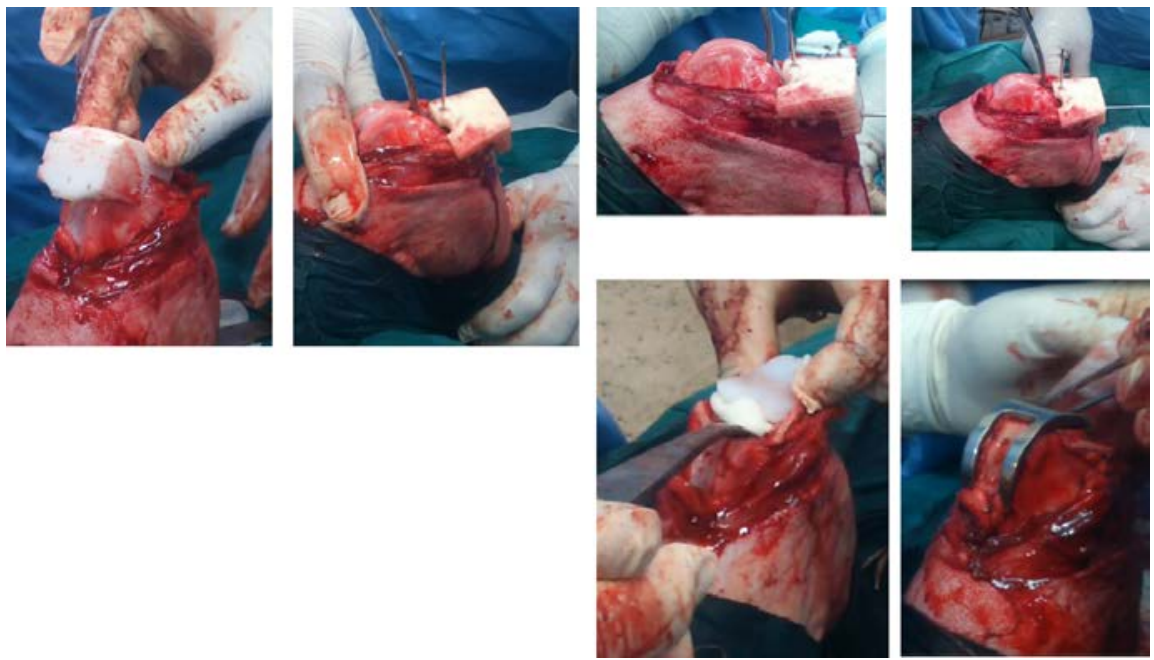

Figure (11): intraoperative photos showing the positioning and fixation of patient specific template and implant positioning after performing surgery. 